\title{
Long-term risk of subsequent ipsilateral lesions after surgery with or without radiotherapy for ductal carcinoma in situ of the breast
}

\author{
Maartje van Seijen (D) ${ }^{1}$, Esther H. Lips (D) ${ }^{1}$, Liping Fu' ${ }^{1}$, Daniele Giardiello ${ }^{1}$, Frederieke van Duijnhoven ${ }^{2}$, Linda de Munck (D) ${ }^{3}$, \\ Lotte E. Elshof ${ }^{4}$, Alastair Thompson ${ }^{5}$, Elinor Sawyer (iD) ${ }^{6}$, Marc D. Ryser ${ }^{7,8}$, E. Shelley Hwang ${ }^{9}$, Marjanka K. Schmidt ${ }^{1,10}$, \\ Paula H. M. Elkhuizen ${ }^{11}$, Grand Challenge PRECISION Consortium, Jelle Wesseling (ID ${ }^{12,13,15^{凶}}$ and Michael Schaapveld $^{14,15}$
}

(c) The Author(s) 2021

BACKGROUND: Radiotherapy (RT) following breast-conserving surgery (BCS) for ductal carcinoma in situ (DCIS) reduces ipsilateral breast event rates in clinical trials. This study assessed the impact of DCIS treatment on a 20-year risk of ipsilateral DCIS (iDCIS) and ipsilateral invasive breast cancer (ilBC) in a population-based cohort.

METHODS: The cohort comprised all women diagnosed with DCIS in the Netherlands during 1989-2004 with follow-up until 2017. Cumulative incidence of iDCIS and IIBC following BCS and BCS + RT were assessed. Associations of DCIS treatment with iDCIS and ilBC risk were estimated in multivariable Cox models.

RESULTS: The 20-year cumulative incidence of any ipsilateral breast event was $30.6 \%$ (95\% confidence interval (Cl): $28.9-32.6)$ after BCS compared to $18.2 \%(95 \% \mathrm{Cl} 16.3-20.3)$ following BCS + RT. Women treated with BCS compared to BCS + RT had higher risk of developing iDCIS and ilBC within 5 years after DCIS diagnosis (for iDCIS: hazard ratio (HR) age $<503.2(95 \% \mathrm{Cl} 1.6-6.6)$; $\mathrm{HR}_{\mathrm{age}} \geq 503.6$ (95\% Cl 2.6-4.8) and for ilBC: $\mathrm{HR}_{\text {age }<50} 2.1$ (95\% Cl 1.4-3.2); $\mathrm{HR}_{\text {age }} \geq 504.3$ (95\% Cl 3.0-6.0)). After 10 years, the risk of iDCIS and ilBC no longer differed for BCS versus BCS + RT (for iDCIS: $\mathrm{HR}_{\mathrm{age}<50} 0.7$ (95\% Cl 0.3-1.5); $\mathrm{HR}_{\mathrm{age}} \geq 500.7(95 \% \mathrm{Cl} 0.4-1.3)$ and for ilBC: $\mathrm{HR}_{\text {age }<50} 0.6$ (95\% Cl 0.4-0.9); $\mathrm{HR}_{\text {age } \geq 50} 1.2$ (95\% Cl 0.9-1.6)).

CONCLUSION: RT is associated with lower iDCIS and ilBC risk up to 10 years after BCS, but this effect wanes thereafter.

British Journal of Cancer (2021) 125:1443-1449; https://doi.org/10.1038/s41416-021-01496-6

\section{INTRODUCTION}

Since the introduction of population-based mammography breast cancer screening in the 1990s, ductal carcinoma in situ (DCIS) comprises $\sim 15 \%$ of all newly diagnosed neoplastic breast lesions $[1,2]$. DCIS is considered a non-obligate precursor of invasive breast cancer (IBC) and consists of neoplastic epithelial cells confined to the ductal system of the mammary gland. Because of its potential to become invasive, patients diagnosed with DCIS are usually treated for IBC with a mastectomy or with breastconserving surgery (BCS) often followed by radiotherapy (RT) to the whole breast (RT). DCIS itself, however, is not life-threatening, and these treatment strategies by definition lead to overtreatment for lesions, which would not progress to IBC within the lifespan of that patient $[3,4]$.

RT as an adjunct to BCS as a treatment for DCIS was evaluated in several clinical trials (NSABP B17, EORTC 10853, SweDCIS, UK/ANZ), and a meta-analysis demonstrated a $15 \%$ absolute 10 -year risk reduction of both subsequent ipsilateral DCIS (iDCIS) and ipsilateral IBC (iIBC) lesions for BCS + RT versus BCS only, without effect on breast cancer-specific survival and overall survival [5-9]. However, how these trial data translate into a reduction of ipsilateral breast events in large, population-based patient cohorts in the longer term is unclear. We previously showed an absolute risk for ilBC of $15.4 \%$ for patients treated with BCS only compared

\footnotetext{
${ }^{1}$ Division of Molecular Pathology, The Netherlands Cancer Institute-Antoni van Leeuwenhoek Hospital, Amsterdam, The Netherlands. ${ }^{2}$ Department of Surgery, The Netherlands Cancer Institute-Antoni van Leeuwenhoek Hospital, Amsterdam, The Netherlands. ${ }^{3}$ Department of Research and Development, Netherlands Comprehensive Cancer Organisation, Utrecht, The Netherlands. ${ }^{4}$ Department of radiology, Meander Medical Centre, Amersfoort, The Netherlands. ${ }^{5}$ Dan L Duncan Comprehensive Cancer Centre, Baylor College of Medicine, Houston, TX, USA. ${ }^{6}$ Division of Cancer Studies, King's College London, Comprehensive Cancer Centre, Guy's Hospital, London, UK. ${ }^{7}$ Department of Population Health Sciences, Duke University, Durham, NC, USA. ${ }^{8}$ Department of Mathematics, Duke University, Durham, NC, USA. ${ }^{9}$ Department of Surgery, Duke University, Durham, NC, USA. ${ }^{10}$ Department of clinical genetics, Leiden University Medical Center, Leiden, The Netherlands. ${ }^{11}$ Department of radiotherapy, The Netherlands Cancer Institute-Antoni van Leeuwenhoek Hospital, Amsterdam, The Netherlands. ${ }^{12}$ Department of pathology, The Netherlands Cancer Institute-Antoni van Leeuwenhoek Hospital, Amsterdam, The Netherlands. ${ }^{13}$ Department of Pathology, Leiden University Medical Center, Leiden, The Netherlands. ${ }^{14}$ Division of Psychosocial Research and Epidemiology, The Netherlands Cancer Institute-Antoni van Leeuwenhoek Hospital, Amsterdam, The Netherlands. ${ }^{15}$ These authors contributed equally: Jelle Wesseling, Michael Schaapveld. A full list of Grand Challenge PRECISION Consortium Steering Group and their affiliations appears in the Supplementary Information. ${ }^{凶}$ email: j.wesseling@nki.nl
}

Received: 4 December 2020 Revised: 20 May 2021 Accepted: 9 July 2021

Published online: 18 August 2021 
to $8.8 \%$ for patients treated with $\mathrm{BCS}+\mathrm{RT}$ at 15 years after diagnosis in a cohort with nationwide coverage [10]. Importantly, we observed that iIBC risk no longer appeared to differ in the interval beyond 10 years of follow-up when comparing women treated with BCS only to those treated with BCS + RT. Information regarding subsequent in situ lesions was lacking in our previous study. We now assess the very long-term risk of both iDCIS and ilBC after a diagnosis of primary DCIS, extending median follow-up of $>5$ years, and assess associations with initial DCIS treatment by age at DCIS diagnosis and elapsed time since diagnosis.

\section{METHODS \\ Data collection}

Our cohort comprises all women diagnosed with primary pure DCIS in the Netherlands between January 1, 1989 and December 31, 2004 [10]. Diagnoses of subsequent ilBC lesions were derived from the Netherlands Cancer Registry (NCR) as well as through linkage of the NCR database with the nationwide registry of histology and cytopathology in the Netherlands (PALGA). Subsequent iDCIS lesions are not registered within the NCR and therefore identification is solely based on pathology reports provided by the PALGA registry. iDCIS was defined as any ipsilateral ductal carcinoma in situ lesion including microinvasive growth $<1 \mathrm{~mm}$ at least 3 months after diagnosis of the index DCIS; ilBC was defined as any ipsilateral invasive breast lesion (corresponding to DCIS $>1 \mathrm{~mm}$ microinvasive growth) diagnosed at least 3 months after diagnosis of the index DCIS. Follow-up for both NCR and PALGA has been completed until January 1,2017. Initial treatment was categorised into three groups: BCS alone (BCS only), BCS with additional whole breast RT $(B C S+R T)$ or mastectomy (independent of subsequent RT). Chemotherapy and endocrine therapy were almost never administered to women with DCIS in the Netherlands during the time of the cohort accrual, and patients who received chemotherapy or endocrine therapy for DCIS were excluded $(n=$ 123). For patients treated with mastectomy, information of iDCIS recurrences was not collected, because a priori we expected these to be negligibly low. Intercurrent mastectomies were defined as mastectomies of the ipsilateral breast $\geq 3$ months after primary DCIS diagnosis and applied for other reasons than our events of interest (iDCIS or ilBC) as identified from pathology reports provided by the PALGA registry. As the Netherlands has a universal health care system for all inhabitants, all women diagnosed with DCIS had equal access to treatment. In this paper, subsequent ipsilateral lesions are referred to as 'recurrence', although we do not know whether these lesions are biologically related to the primary DCIS or represent independent secondary primaries.

\section{Statistical analyses}

Time at risk started at the date of primary DCIS diagnosis and ended the date of the first event of interest (iDCIS or IIBC), date of death, emigration or January 1,2017 , whichever came first. If the laterality of a subsequent
iDCIS was unknown, this resulted in censoring at the date of iDCIS $(n=10)$. The cumulative incidence of $\mathrm{iDCIS}$, ilBC and the combination of iDCIS and ilBC was estimated using the Aalen-Johanson estimator with death as the only competing risk and emigration as a censoring event. iDCIS or IIBC was not used as a competing event nor as a censoring event when evaluating the risk of the other. In the cumulative incidence analysis for iIBC, an intercurrent mastectomy was included as time-dependent co-variable, in which patients with an intercurrent mastectomy contributed personal time to the mastectomy group from the date of that intercurrent mastectomy. An intercurrent mastectomy (independent of the reason for the mastectomy) is a censoring event in all the iDCIS analyses and in the Cox analyses for ilBC. For the cumulative incidence analyses, Grays' sample test [11] was used to assess the differences between treatments; intercurrent mastectomies were not taken into account.

Multivariable Cox proportional hazard analysis was used to examine the effects of treatment strategies on IDCIS and IIBC risk. Attained age was used as a timescale. The proportional hazard assumption was assessed using residualbased and graphical methods. Because the hazard ratios (HRs) for treatment were non-proportional with time since treatment, the models for iDCIS and ilBC risk were stratified by time since treatment, using intervals of 0-4, 5-9 and $\geq 10$ years after diagnosis and an interaction term for treatment and time since treatment, using the above intervals, was added to the models [12]. In addition, the HRs for treatment differed with age at diagnosis $\left(p_{\text {interaction }}<0.001\right)$. Using the Aikake information criterion, the ilBC model demonstrated the best fit when age at DCIS diagnosis was fitted as a dichotomous categorical variable ( $<50$ versus $\geq 50$ years old) and an age-treatment interaction term was added to the model. For iDCIS, the best fit model was achieved by adjusting for age at DCIS diagnosis as a continuous variable. To keep the models for iDCIS and iIBC comparable, we, however, included age as a dichotomous categorical variable ( $<50$ versus $\geq 50$ years old), while also including an age-treatment interaction term, although for iDCIS this age-treatment interaction was non-significant $\left(p_{\text {interaction }}=0.06\right)$.

The association of histological grade of the primary DCIS with iDCIS and ilBC risks was evaluated only among patients diagnosed in the period 1999-2004, as information on DCIS grade was incomplete before 1999. In the analysis of iDCIS risk among patients diagnosed in 1999-2004, the proportional hazards assumption was not violated and no interaction term for treatment and time since treatment was included and age neither modified the effect of treatment.

All analyses were performed in open source software $\mathrm{R}$ version 3.5.1 using the 'survival' and 'etm' packages [13].

\section{RESULTS}

The study cohort comprised 10,045 women, of whom 2647 (26\%) received BCS only, 2604 (26\%) received BCS + RT and 4794 (48\%) underwent mastectomy as the primary treatment. Additional patient characteristics are summarised in Table 1. The median

Table 1. Patient characteristics.

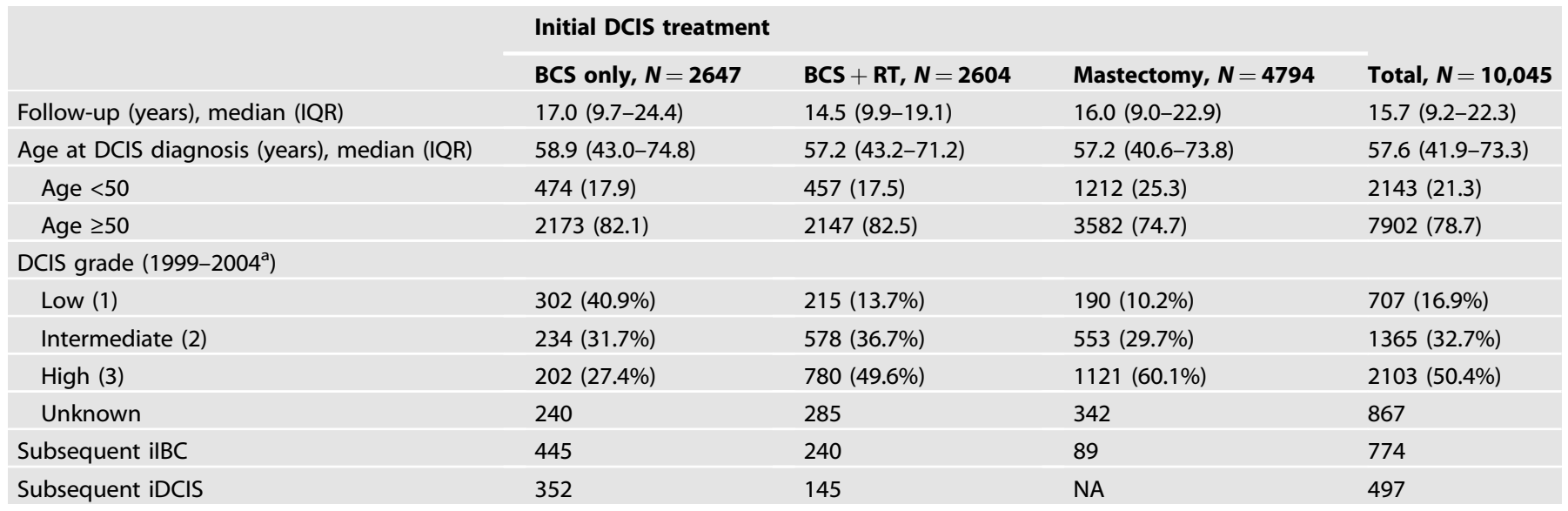

IIBC ipsilateral invasive breast cancer, $i D C I S$ ipsilateral ductal carcinoma in situ, $B C S$ breast-conserving surgery, $R T$ radiotherapy, $N$ number, $I Q R$ interquartile range, DCIS ductal carcinoma in situ, NA not available.

a Data on grade are presented for patients diagnosed with primary DCIS from 1999 to $2004(n=5042)$. 
follow-up was 15.7 years (interquartile range 9.2-22.3 years). During follow-up, a total of 774 (7.7\%) ilBC and 497 (4.9\%) iDCIS lesions were identified. The 10- and 20-year cumulative incidence of subsequent ipsilateral breast disease (iDCIS or ilBC) for women treated with BCS only was $24.6 \%$ (95\% confidence interval $(\mathrm{Cl})$ : 23.0-26.3) and 30.6\% (95\% Cl 28.9-32.6), respectively, whereas for women treated with BCS + RT, the cumulative incidence was $9.6 \%$ $(95 \% \mathrm{Cl} 8.6-10.8)$ and $18.2 \%(95 \% \mathrm{Cl} 16.3-20.3)$ at 10 and 20 years, respectively (Fig. 1). The competing risk, death, varied for the different treatment strategies between 8.7 and $14.7 \%$ after 10 years and between 26.8 and $35.2 \%$ after 20 years since DCIS diagnosis (Supplementary Fig 1).

\section{Subsequent iDCIS risk}

Among patients treated with BCS only, 352 iDCIS occurred, compared to $145 \mathrm{iDCIS}$, among patients treated with BCS + RT. Most iDCIS occurred within the first 10 years of follow-up, with only 19 patients developing a late iDCIS (10 years or more after their initial DCIS diagnosis) after BCS only and 27 after BCS + RT (Supplementary Table 1). For women treated with BCS only, the 10 - and 20-year cumulative incidence of iDCIS was $13.0 \%(95 \% \mathrm{CI}$ $11.8-14.4)$ and $13.9 \%(95 \% \mathrm{Cl} 11.6-15.3)$, respectively, versus $4.6 \%$ $(95 \% \mathrm{Cl} 3.9-5.5)$ and $6.7 \%(95 \% \mathrm{Cl} 5.5-8.1)$, respectively, for women treated with BCS + RT (Fig. 1 and Supplementary Table 1).

Women $<50$ years treated with BCS only had 3.2 times higher HR $(95 \% \mathrm{Cl} 1.6-6.6)$ for iDCIS in the first 5 years after diagnosis compared to women treated with $\mathrm{BCS}+\mathrm{RT}$, while women $\geq 50$ years treated with BCS only had a 3.6 times higher HR for iDCIS (95\% Cl 2.6-4.8) than women treated with BCS + RT (Table 2). The $\mathrm{HR}$ to develop iDCIS among patients treated with BCS only compared to $\mathrm{BCS}+\mathrm{RT}$ in the interval 5-9 years after primary DCIS was $2.5(95 \% \mathrm{Cl} 1.1-5.3)$ for women $<50$ years and $2.7(95 \% \mathrm{Cl}$ 1.8-4.1) for women $\geq 50$ years, and risks no longer differed between patients treated with BCS only compared to BCS + RT from 10 years after initial DCIS (Table 2). Women diagnosed between 1999 and 2004 had a slightly lower risk of developing iDCIS compared to women diagnosed between 1989 and 1998 (HR 0.9; 95\% Cl 0.7-1.0).

Among all women diagnosed with primary DCIS between 1999 and 2004, women with grade 1 DCIS had half the risk (HR 0.5; 95\% $\mathrm{Cl}$ 0.3-0.8) of iDCIS compared to women with grade 2 lesions (Supplementary Table 2). iDCIS risk did not differ for women with grade 3 lesions compared to those with grade 2 lesions. As 58 patients developed a subsequent iDCIS between 3 and 6 months after DCIS diagnosis, as a sensitivity analysis, we also assessed the risk of iDCIS starting follow-up at 6 months after DCIS diagnosis. Although the HRs slightly increased, particularly for women $<50$ years, the direction of effects remained the same (see Supplementary Table 3).

\section{Subsequent ilBC risk}

Among patients treated with BCS only, the 10- and 20-year cumulative incidence of ilBC was $13.9 \%(95 \% \mathrm{Cl} 11.7-14.3)$ and $19.1 \%(95 \% \mathrm{Cl} 17.5-20.8)$, respectively. The 10- and 20-year cumulative incidence was $5.2 \%(95 \% \mathrm{Cl} 4.4-6.2)$ and $12.1 \%(95 \%$ $\mathrm{Cl}$ 10.5-14.0), respectively, in patients treated with BCS + RT and $1.1 \%(95 \% \mathrm{Cl} 0.9-1.5)$ and $1.9 \%(95 \% \mathrm{Cl} 1.6-2.4)$, respectively, in patients treated with mastectomy (Fig. 1 and Supplementary Table 1). Women $<50$ years diagnosed with DCIS between 1999 and 2004 and treated with BCS + RT showed continuously lower absolute iIBC risks compared to those treated with BCS only (Fig. 2). In contrast, women $<50$ years diagnosed between the period 1989 and 1998 had approximately similar cumulative incidences after either BCS only or BCS + RT treatment from 10 years or more after DCIS diagnosis.

In women $<50$ years at DCIS diagnosis, the HR for ilBC was 2.1 times $(95 \% \mathrm{Cl} 1.4-3.2)$ higher in the first 5 years after diagnosis among those treated with BCS only compared to women treated a iDCIS + ilBC
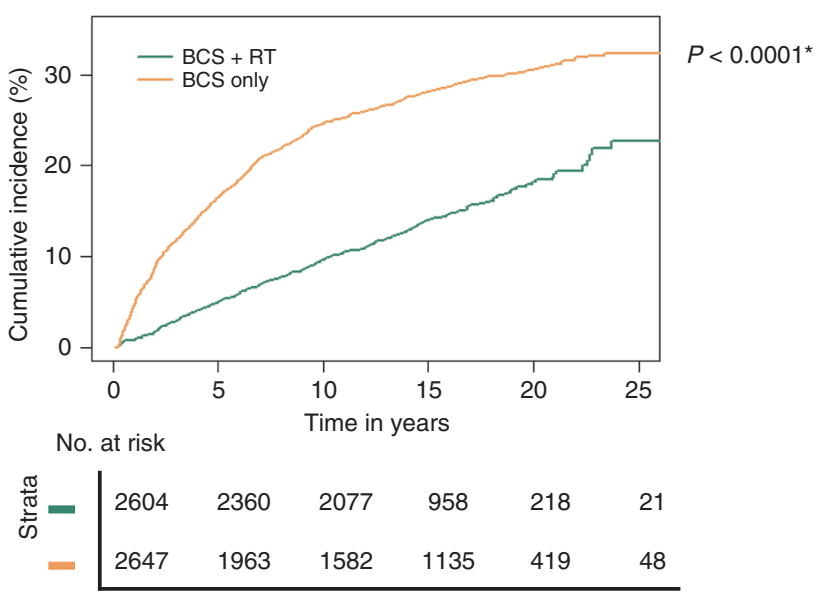

b iDCIS

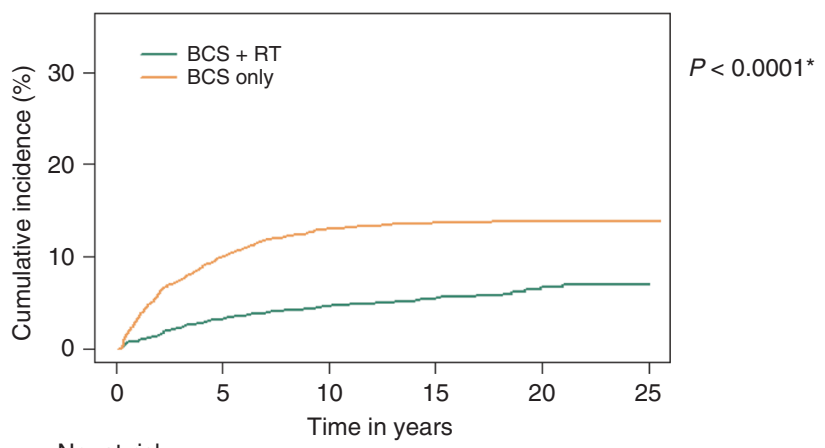

No. at risk

\begin{tabular}{|c|c|c|c|c|c|c|}
\hline 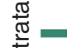 & 2604 & 2394 & 2153 & 1029 & 240 & 28 \\
\hline 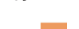 & 2647 & 2087 & 1789 & 1330 & 508 & 65 \\
\hline
\end{tabular}

C ilBC

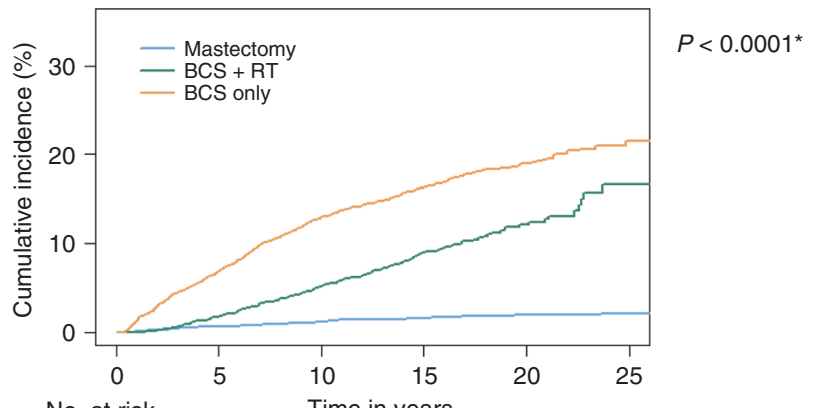

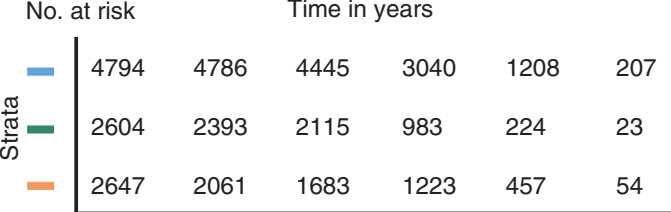

Fig. 1 Cumulative incidence with death as the competing risk by treatment strategy. a In situ and invasive recurrences, b iDCIS only and $\mathbf{c}$ invasive recurrences only. ${ }^{*} P$ values are based on Grays' $K$ sample test.

with BCS + RT; the HR for ilBC was even 4.3 times (95\% CI 3.0-6.0) higher for women $\geq 50$ years treated with $\mathrm{BCS}$ only within the first 5 years after treatment compared to BCS + RT (Table 2). The risk of developing an ilBC no longer differ from 5 years after DCIS 
Table 2. Multivariate Cox analysis to estimate the association of treatment with the risk of subsequent iDCIS and ilBC.

\begin{tabular}{|c|c|c|c|c|c|c|}
\hline \multirow[b]{2}{*}{ Age at DCIS (years) } & \multirow[b]{2}{*}{ Time since DCIS (years) } & \multirow[b]{2}{*}{ Treatment } & \multicolumn{2}{|l|}{ iDCIS } & \multicolumn{2}{|l|}{ ilBC } \\
\hline & & & Events $(N) /$ at risk $(N)$ & HR $(95 \% \mathrm{CI})$ & Events $(N)$ /at risk $(N)$ & HR $(95 \% \mathrm{Cl})$ \\
\hline \multirow[t]{7}{*}{$<50(N=2143)$} & \multirow[t]{2}{*}{$0-5$} & $\mathrm{BCS}+\mathrm{RT}$ & $15 / 457$ & Ref. & $17 / 457$ & Ref. \\
\hline & & Mastectomy ${ }^{\mathrm{a}}$ & - & - & $19 / 1212$ & $0.4(0.2-0.6)$ \\
\hline & \multirow[t]{2}{*}{$5-10$} & $\mathrm{BCS}+\mathrm{RT}$ & $8 / 419$ & Ref. & $22 / 412$ & Ref. \\
\hline & & BCS only & $12 / 386$ & $2.5(1.1-5.3)$ & $23 / 379$ & $1.0(0.7-1.5)$ \\
\hline & \multirow[t]{3}{*}{$\geq 10$} & $\mathrm{BCS}+\mathrm{RT}$ & $17 / 383$ & Ref. & $38 / 363$ & Ref. \\
\hline & & BCS only & $4 / 353$ & $0.7(0.3-1.5)$ & $36 / 331$ & $0.6(0.4-0.9)$ \\
\hline & & Mastectomy ${ }^{\mathrm{a}}$ & - & - & $17 / 1108$ & 0.1 (0.1v0.2) \\
\hline \multirow[t]{6}{*}{$\geq 50(N=7902)$} & \multirow[t]{2}{*}{$0-5$} & $\mathrm{BCS}+\mathrm{RT}$ & $69 / 2147$ & Ref. & $29 / 2147$ & Ref. \\
\hline & & BCS only & $201 / 2173$ & $3.6(2.6-4.8)$ & $137 / 2173$ & $4.3(3.0-6.0)$ \\
\hline & $5-10$ & Mastectomy ${ }^{\mathrm{a}}$ & - & - & $9 / 3314$ & $0.1(0.1-0.2)$ \\
\hline & \multirow[t]{3}{*}{$\geq 10$} & $\mathrm{BCS}+\mathrm{RT}$ & $10 / 1769$ & Ref. & $70 / 1751$ & Ref. \\
\hline & & BCS only & $15 / 1436$ & $0.7(0.4-1.3)$ & $94 / 1352$ & $1.2(0.9-1.6)$ \\
\hline & & Mastectomy ${ }^{\mathrm{a}}$ & - & - & $17 / 2957$ & $0.1(0.1-0.1)$ \\
\hline
\end{tabular}

$H R$ hazard ratio, 95\% Cl 95\% confidence interval, Ref. reference category, BCS breast-conserving surgery, RT radiotherapy, iDCIS ipsilateral ductal carcinoma in situ, ilBC ipsilateral invasive breast cancer, DCIS ductal carcinoma in situ.

anformation regarding mastectomy treatment was not available for iDCIS. Attained age was used as a primary timescale, adjusted for a period of initial DCIS diagnosis (1989-1998 versus 1999-2004) and age at DCIS diagnosis ( $<50$ versus $\geq 50$ ), including an age-treatment interaction term.

diagnosis for women $<50$ years compared to those treated with BCS only or with BCS + RT (HR 1.0; 95\% Cl 0.7-1.5). While for women $\geq 50$ years, this risk did no longer differ from 10 years after DCIS diagnosis (HR 1.2; $95 \% \mathrm{Cl} 0.9-1.6)$. Women treated with a mastectomy had a much lower risk of developing ilBC compared to women treated with BCS, irrespective of age at diagnosis or time since DCIS treatment (Table 2). Women diagnosed with primary DCIS between 1999 and 2004 had a slightly lower risk of developing ilBC compared to women diagnosed between 1989 and 1999 (HR 0.8; 95\% Cl 0.6-0.9).

Inclusion of histological grade in the analysis did not affect the association of DCIS treatment with ilBC risk $\left(\mathrm{HR}_{\mathrm{age}} \geq 50\right.$ for $\mathrm{BCS}$ only versus $\mathrm{BCS}+\mathrm{RT}$ in year $1-5: 4.8 ; 95 \% \mathrm{Cl} 2.7-8.5)$ for a model including grade and $4.8(95 \% \mathrm{Cl} 2.7-8.6)$ for a model without grade, see Supplementary Table 4 for all estimates) and grade did not modify the association of initial treatment with ilBC risk $\left(p_{\text {interaction }}=0.3\right)$. As information regarding comorbidities was unavailable, a sensitivity analysis was performed excluding patients $\geq 70$ years, assuming that patients $\geq 70$ years would be most at risk for comorbidities. We found slightly lower risk in the first 5 years after DCIS diagnosis for women $\geq 50$ years treated with $B C S$ only versus $B C S+R T$ (HR 3.6; 95\% Cl 2.7-4.8) compared to the analysis including all women (HR 4.3; 95\% Cl 3.0-6.0) (Supplementary Table 5).

\section{DISCUSSION}

In this population-based study among 10,045 women treated for DCIS, we showed that patients treated with BCS only had an absolute risk of $14 \%$ to develop iDCIS and of $19 \%$ to develop iIBC at 20 years after treatment, while for BCS + RT, these risks were $7 \%$ and $12 \%$, respectively. Furthermore, RT is most strongly associated with lower recurrence risks in the first decade after DCIS diagnosis. iDCIS predominantly occurred in the first 10 years after primary DCIS. Finally, the rate of iIBC recurrences did no longer differ between women treated with BCS only versus BCS + RT from 5 years after DCIS diagnosis in women $<50$ years and from 10 years after DCIS diagnosis in women $\geq 50$ years at primary DCIS.

Our study has some limitations. Firstly, margin status, tumour size and information on initiation and completion of RT were not available for our patients while grade was not routinely scored in the period covered by our study, and, also because of (high) interobserver variability $[14,15]$, may not have been very reliably assessed. ER and HER2 status are not routinely determined for DCIS lesions in the Netherlands, because it has no therapeutic consequences.

We had no information on comorbidities. In addition, our cohort might not be completely representative of DCIS patients nowadays. The patients in our cohort were diagnosed and treated sometimes decades ago, and pre-operative work-up and RT techniques have evolved over time.

However, the strengths of this study are that it is truly population-based, covering the whole of the Netherlands, with complete information on initial treatment and reliable information on intercurrent mastectomies combined with long and complete follow-up for both in situ IBC and IBC, as well as vital status. Although we defined iDCIS and IIBC as subsequent ipsilateral recurrences $\geq 3$ months after DCIS diagnosis, starting follow-up at 6 months after diagnosis could slightly reduce the chance of counting re-excisions as iDCIS events.

Nonetheless, our data clearly show that late in situ recurrences, $\geq 10$ years after DCIS diagnosis, rarely developed, while the incidence of iIBC continued to rise over time irrespective of initial treatment. This is concordant with the SweDCIS trial [16] and with the Vermont cohort [17], which both reported few iDCIS occurrences after 5 years of follow-up.

An explanation for this plateau in risk of subsequent iDCIS lesions after 10 years might be that recurrent DCIS lesions were less detected after 10 years either due to the fact that patients were discharged from routine surveillance or were no longer 

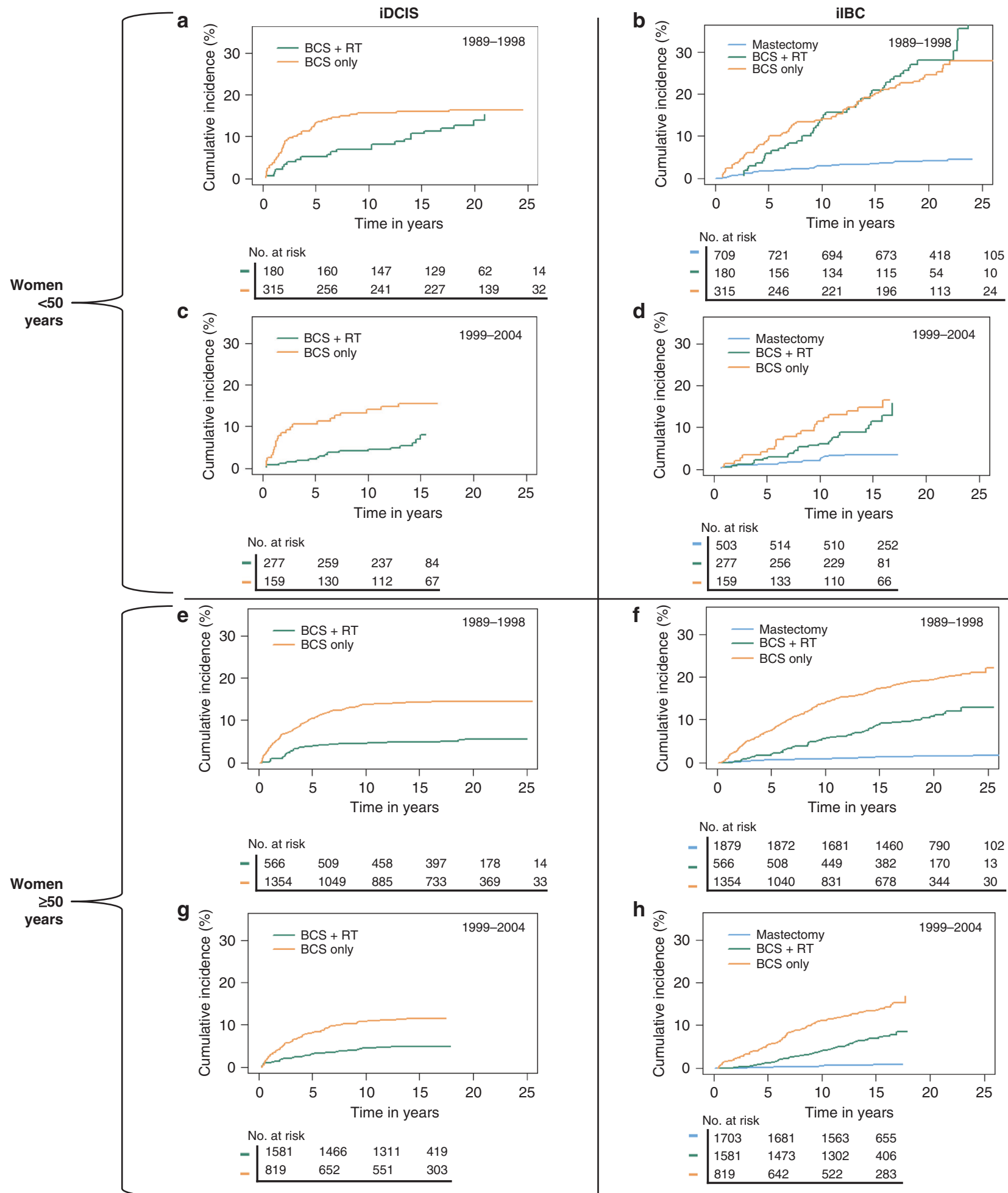

\begin{tabular}{|c|c|c|c|}
\hline 503 & 514 & 510 & 252 \\
\hline 277 & 256 & 229 & 81 \\
\hline 159 & 133 & 110 & 66 \\
\hline
\end{tabular}
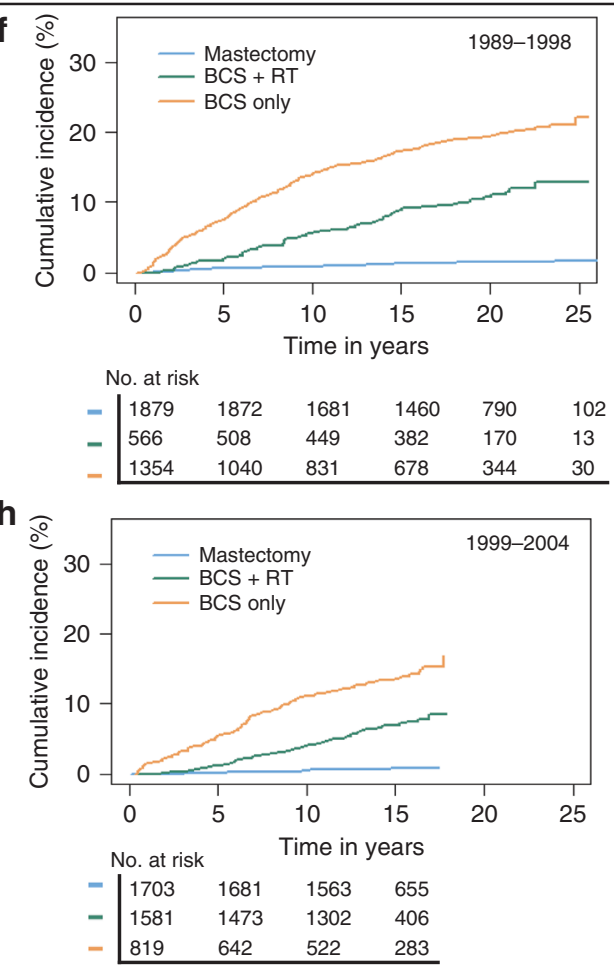

Fig. 2 Cumulative incidence of iDCIS and iIBC with death as the competing risk splitted for period and age. Cumulative incidence with death as the competing risk in a iDCIS risk of women $<50$ years diagnosed between 1989 and 1998 for primary DCIS, $\mathbf{b}$ ilBC risk of women $<50$ years diagnosed between 1989 and 1998 for primary DCIS, c iDCIS risk of women <50 years diagnosed between 1999 and 2004 for primary DCIS and d ilBC risk women <50 years diagnosed between 1999 and 2004 for primary DCIS, e iDCIS risk of women $\geq 50$ years diagnosed between 1989 and 1998, $\mathbf{f}$ ilBC risk of women $\geq 50$ years diagnosed between 1989 and 1998, $\mathbf{g}$ iDCIS risk of women $\geq 50$ years diagnosed between 1999 and 2004 and $\mathbf{h}$ ilBC risk of women $\geq 50$ years diagnosed between 1999 and 2004.

within the age range invited for the population breast cancer screening programme. Alternatively, the lack of in situ recurrences after 10 years may reflect the biology of these DCIS lesions, which would suggest that almost all subsequent iDCIS lesions originate from residual primary DCIS. This is supported by the high frequency of clonal relatedness of iDCIS to primary DCIS, reported to be $82 \%$ by Waldman et al. [18], while Shah et al. [19] even reported complete clonal relatedness of iDCIS to primary DCIS. 
Within our consortium, PREvent ductal Carcinoma In Situ Invasive Overtreatment Now (PRECISION) initiative [20], we are conducting genomic studies to determine the clonal relatedness of in situ recurrences to the primary DCIS in order to better understand the relationship between the initial DCIS diagnosis and subsequent breast events.

RT is associated with a lower risk of iDCIS and iIBC, particularly in the first 10 years after the initial DCIS diagnosis. This is in line with a prior meta-analysis that showed that RT reduced the absolute 10 -year risk by $15 \%(28.1 \%$ any recurrence in BCS-only group versus $12.9 \%$ in $\mathrm{BCS}+\mathrm{RT}$ group [6]) and with several cohort studies, which all showed that RT reduced breast events after RT in addition to BCS $[17,21-23]$. However, our analysis also showed that 10 years or more after DCIS diagnosis, the incidence of new ilBC is approximately similar in the BCS-only and BCS + RT group (Fig. 1 and Supplementary Table 1). This is consistent with the results of Rakovitch et al. [24], who showed lower risks of second breast events with increasing follow-up time after DCIS diagnosis. Since extensive clonal diversity is generated by mutations gradually evolving over time [25], it becomes more likely that newly developed tumours represent an independent second primary tumour $>10$ years after initial DCIS. However, to our knowledge, the association of follow-up time with clonal relatedness between primary DCIS and subsequent lesions has not yet been assessed. In addition, we cannot exclude the possibility that RT may induce (secondary) invasive breast tumours, which may become apparent long after exposure to RT. A meta-analysis by Akdeniz et al. did demonstrate a slightly increased risk of contralateral breast cancer after RT mainly in breast cancer patients treated $<45$ years of age [26].

Women $<50$ years diagnosed with primary DCIS between 1989 and 1998 had similar absolute late ilBC risk irrespective of treatment with BCS only or BCS + RT (Fig. 2). The SweDCIS trial neither showed a long-term beneficial effect of RT following BCS on ilBC risk in young women ( $<52$ years) [16]. In our models, we split age at 50 years because the Dutch nationwide breast cancer screening starts at the age of 50 years and thus a diagnosis of primary DCIS in women $<50$ years is rarely based on breast screening. These women may present with a different type of DCIS, including more frequent symptomatic presentation (i.e. a lump), and/or may be diagnosed in the light of familial genetic susceptibility syndromes, which may be accompanied by an increased risk of ilBC. In addition, some studies [24, 27] showed that younger patients, in general, have a higher risk of invasive recurrences compared to older patients. However, Ryser et al. [3] did not find that ilBC risks were different between women aged $<50$ and $\geq 50$ years, although this study was not powered to examine age differences. Therefore, we would be cautious against the interpretation that younger women benefit less from RT.

This large population-based DCIS cohort provides insight into the long-term risks of ipsilateral breast recurrences in women treated for DCIS. As DCIS is not a life-threatening disease, our ultimate goal should be to de-escalate treatment. There are ongoing efforts to determine whether molecular profiles of DCIS, such as Oncotype DX DCIS score [28] or DCISionRT signature [29], could support the selection of women in whom RT could be safely omitted. Furthermore, three ongoing clinical trials (LORIS [30], LORD [31] and COMET [32] trials) currently randomise between active surveillance and conventional treatment to omit therapy for women with low-risk DCIS. Understanding the dynamics of longterm residual breast cancer risk following treatment of DCIS contributes to the understanding of this disease and finally to reducing overtreatment.

\section{DATA AVAILABILITY}

The data generated and analysed during this study will be available from the corresponding author upon reasonable request.

\section{REFERENCES}

1. Netherlands Comprehensive Cancer Organisation (IKNL). DCIS incidence trends over time. The Netherlands. https://iknl.nl/nkr.

2. Howlader N, Noone A, Krapcho M, Miller D, Brest A, Yu M, et al. SEER Cancer Statistics Review, 1975-2017. Bethesda: National Cancer Institute; 2017. https://seer.cancer.gov/csr/1975_2017/.

3. Ryser MD, Weaver DL, Zhao F, Worni M, Grimm LU, Gulati R, et al. Cancer outcomes in DCIS patients without locoregional treatment. J Natl Cancer Inst. 2019;111:1-9. http://www.ncbi.nlm.nih.gov/pubmed/30759222/.

4. Erbas B, Provenzano E, Armes J, Gertig D. The natural history of ductal carcinoma in situ of the breast: a review. Breast Cancer Res Treat. 2006;97:135-44.

5. Fisher B, Dignam J, Wolmark N, Mamounas E, Costantino J, Poller W, et al. Lumpectomy and radiation therapy for the treatment of intraductal breast cancer: findings from National Surgical Adjuvant Breast and Bowel Project B-17. J Clin Oncol. 1998;16:441-52. http://www.ncbi.nlm.nih.gov/pubmed/9469327.

6. Correa C, McGale P, Taylor C, Wang Y, Clarke M, Davies C, et al. Overview of the randomized trials of radiotherapy in ductal carcinoma in situ of the breast. J Natl Cancer Inst Monogr. 2010;2010:162-77.

7. Bijker N, Meijnen P, Peterse JL, Bogaerts J, Van Hoorebeeck I, Julien J-P, et al. Breast-conserving treatment with or without radiotherapy in ductal carcinomain-situ: ten-year results of European Organisation for Research and Treatment of Cancer Randomized Phase III Trial 10853-a study by the EORTC Breast Cancer Cooperative Group and. J Clin Oncol. 2006;24:3381-7. http://ascopubs.org/doi/ 10.1200/JCO.2006.06.1366.

8. Emdin SO, Granstrand B, Ringberg A, Sandelin K, Arnesson L-GG, Nordgren H, et al. SweDCIS: Radiotherapy after sector resection for ductal carcinoma in situ of the breast. Results of a randomised trial in a population offered mammography screening. Acta Oncol. 2006;45:536-43. http://www.ncbi.nlm.nih.gov/pubmed/ 16864166

9. Houghton J, George WD, Cuzick J, Duggan C, Fentiman IS, Spittle M, et al. Radiotherapy and tamoxifen in women with completely excised ductal carcinoma in situ of the breast in the UK, Australia, and New Zealand: randomised controlled trial. Lancet. 2003;362:95-102. http://www.ncbi.nlm.nih.gov/pubmed/ 12867108.

10. Elshof LE, Schaapveld M, Schmidt MK, Rutgers EJ, van Leeuwen FE, Wesseling J. Subsequent risk of ipsilateral and contralateral invasive breast cancer after treatment for ductal carcinoma in situ: incidence and the effect of radiotherapy in a population-based cohort of 10,090 women. Breast Cancer Res Treat. 2016;159: 553-63.

11. Gray RJ. A class of K-sample tests for comparing the cumulative incidence of a competing risk. Ann Stat. 1988;16:1141-54. http://projecteuclid.org/euclid.aos/ 1176350951.

12. Zhang Z, Geskus RB, Kattan MW, Zhang H, Liu T. Nomogram for survival analysis in the presence of competing risks. Ann Transl Med. 2017;5:47.

13. R Core Team. R: a language and environment for statistical computing. 2018. https://www.r-project.org.

14. Groen EJ, Hudecek J, Mulder L, van Seijen M, Almekinders MM, Alexov S, et al. Prognostic value of histopathological DCIS features in a large-scale international interrater reliability study. Breast Cancer Res Treat. 2020;183:759-70. https://doi. org/10.1007/s10549-020-05816-x.

15. van Dooijeweert C, van Diest PJ, Willems SM, Kuijpers CCHJ, Overbeek LIH, Deckers IAG. Significant inter- and intra-laboratory variation in grading of ductal carcinoma in situ of the breast: a nationwide study of 4901 patients in the Netherlands. Breast Cancer Res Treat. 2018. http://link.springer.com/10.1007/ s10549-018-05082-y.

16. Wärnberg F, Garmo H, Emdin S, Hedberg V, Adwall L, Sandelin K, et al. Effect of radiotherapy after breast-conserving surgery for ductal carcinoma in situ: 20 years follow-up in the randomized SweDCIS trial. J Clin Oncol. 2014;32:3613-8.

17. Sprague BL, Vacek PM, Herschorn SD, James TA, Geller BM, Trentham-Dietz A, et al. Time-varying risks of second events following a DCIS diagnosis in the population-based Vermont DCIS cohort. Breast Cancer Res Treat. 2018;174:0. https://doi.org/10.1007/s10549-018-5048-8.

18. Waldman FM, Devries S, Chew KL, li DHM, Ljung B. Chromosomal alterations in ductal carcinomas in situ. Cancer. 2000;92:313-20.

19. Shah V, Megalios A, Shami R, Sridharan M, Salinas de Souza C, Kumar T, et al. Genomic analysis of paired DCIS and subsequent recurrence to assess clonal relatedness in screen-detected DCIS[abstract]. Proceedings of the 2019 San Antonio Breast Cancer Symposium; 2019 Dec 10-14; San Antonio, TX. Philadelphia (PA): AACR; Cancer Res. 2020;80(4 Suppl):Abstract nr P4-07-04. https://doi.org/10.1158/ 1538-7445.

20. van Seijen M, Lips EH, Thompson AM, Nik-Zainal S, Futreal A, Hwang ES, et al. Ductal carcinoma in situ: to treat or not to treat, that is the question. $\mathrm{Br} J$ Cancer. 2019;121:285-92. https://doi.org/10.1038/s41416-019-0478-6.

21. Thompson AM, Clements K, Cheung S, Pinder SE, Lawrence G, Sawyer E, et al. Management and 5-year outcomes in 9938 women with screen-detected ductal 
carcinoma in situ: the UK Sloane Project On behalf of the Sloane Project Steering Group (NHS Prospective Study of Screen-Detected Non-invasive Neoplasias) 1. Eur J Cancer. 2018;101:210-9. https://doi.org/10.1016/j.ejca.2018.06.027.

22. Mannu GS, Wang Z, Broggio J, Charman J, Cheung S, Kearins O, et al. Invasive breast cancer and breast cancer mortality after ductal carcinoma in situ in women attending for breast screening in England, 1988-2014: population based observational cohort study. BMJ. 2020;369:m1570. http://www.ncbi.nlm.nih.gov/ pubmed/32461218.

23. Shaaban AM, Hilton B, Clements K, Provenzano E, Cheung S, Wallis MG, et al. Pathological features of 11,337 patients with primary ductal carcinoma in situ (DCIS) and subsequent events: results from the UK Sloane Project. Br J Cancer. 2020:1-9. https://doi.org/10.1038/s41416-020-01152-5.

24. Rakovitch E, Sutradhar R, Hallett M, Thompson AM, Gu S, Dumeaux V, et al. The time-varying effect of radiotherapy after breast-conserving surgery for DCIS. Breast Cancer Res Treat. 2019;178:221-30. http://link.springer.com/10.1007/ s10549-019-05377-8.

25. Wang $Y$, Waters J, Leung $M L$, Unruh $A$, Roh W, Shi $X$, et al. Clonal evolution in breast cancer revealed by single nucleus genome sequencing. Nature. 2014;512: 155-60.

26. Akdeniz D, Schmidt MK, Seynaeve CM, McCool D, Giardiello D, van den Broek AJ, et al. Risk factors for metachronous contralateral breast cancer: a systematic review and meta-analysis. Breast. 2019;44:1-14.

27. Solin $\sqcup$, Fourquet A, Vicini FA, Taylor M, Olivotto IA, Haffty B, et al. Long-term outcome after breast-conservation treatment with radiation for mammographically detected ductal carcinoma in situ of the breast. Cancer. 2005;103:1137-46.

28. Rakovitch E, Nofech-Mozes S, Hanna W, Sutradhar R, Baehner FL, Miller DP, et al. Multigene expression assay and benefit of radiotherapy after breast conservation in ductal carcinoma in situ. J Natl Cancer Inst. 2017;109:1-8.

29. Bremer T, Whitworth PW, Patel R, Savala J, Barry T, Lyle S, et al. A biological signature for breast ductal carcinoma in situ to predict radiotherapy benefit and assess recurrence risk. Clin Cancer Res. 2018;24:5895-901.

30. Francis A, Thomas J, Fallowfield L, Wallis M, Bartlett JM, Brookes C, et al. Addressing overtreatment of screen detected DCIS; the LORIS trial. Eur J Cancer. 2015;51:2296-303.

31. Elshof LE, Tryfonidis K, Slaets L, van Leeuwen-Stok AE, Skinner VP, Dif N, et al. Feasibility of a prospective, randomised, open-label, international multicentre, phase III, non-inferiority trial to assess the safety of active surveillance for low risk ductal carcinoma in situ - the LORD study. Eur J Cancer. 2015;51:1497-510. https://doi.org/10.1016/j.ejca.2015.05.008.

32. Hwang ES, Hyslop T, Lynch T, Frank E, Pinto D, Basila D, et al. The COMET (Comparison of Operative versus Monitoring and Endocrine Therapy) trial: a phase III randomised controlled clinical trial for low-risk ductal carcinoma in situ (DCIS). BMJ Open. 2019;9:e026797. http://www.ncbi.nlm.nih.gov/ pubmed/30862637.

\section{ACKNOWLEDGEMENTS}

Not applicable.

\section{AUTHOR CONTRIBUTIONS}

Conception and design: MvS, EHL, LEE, JW and MS. Statistical support: DG, MDR and MKS. Data collection: MvS, LF, LdM, LEE and MS. Data analysis and interpretation:
MvS, EHL, DG, FvD, LdM, AT, LEE, MDR, SH, ES, MKS, PHME, JW and MS. Manuscript writing: all authors. Final approval of manuscript: all authors.

\section{FUNDING}

This work was supported by Cancer Research UK and by KWF Kankerbestrijding (ref. C38317/A24043). This content is solely the responsibility of the authors. The funder had no role in study design, data collection, analysis or interpretation or writing of the manuscript.

\section{COMPETING INTERESTS}

The authors declare no competing interests.

\section{ETHICS APPROVAL AND CONSENT TO PARTICIPATE}

The Central Committee on Research involving Human Subjects determined that this study did not require approval from an ethics committee. The privacy review board of the NCR approved the study.

\section{CONSENT TO PUBLISH}

Not applicable.

\section{ADDITIONAL INFORMATION}

Supplementary information The online version contains supplementary material available at https://doi.org/10.1038/s41416-021-01496-6.

Correspondence and requests for materials should be addressed to J.W

Reprints and permission information is available at http://www.nature.com/ reprints

Publisher's note Springer Nature remains neutral with regard to jurisdictional claims in published maps and institutional affiliations.

\begin{abstract}
Open Access This article is licensed under a Creative Commons Attribution 4.0 International License, which permits use, sharing, adaptation, distribution and reproduction in any medium or format, as long as you give appropriate credit to the original author(s) and the source, provide a link to the Creative Commons license, and indicate if changes were made. The images or other third party material in this article are included in the article's Creative Commons license, unless indicated otherwise in a credit line to the material. If material is not included in the article's Creative Commons license and your intended use is not permitted by statutory regulation or exceeds the permitted use, you will need to obtain permission directly from the copyright holder. To view a copy of this license, visit http://creativecommons. org/licenses/by/4.0/.
\end{abstract}

(c) The Author(s) 2021 